The overall mortality rate was $92 \%$, the duration of hospitalization was between one and 137 days.

Conclusion In our study, it has been seen that NCPAP may not be an effective ventilation strategy in premature infants who are at the limits of viability. The high proportion of chorioamnionitis in this group may affect the ventilation and the following problems. These babies are needed to be care at very special settings.

\section{CLINICAL OUTCOMES OF VERY-LOW-BIRTH-WEIGHT INFANTS WHO RECEIVE NON-INVASIVE VENTILATORY SUPPORT}

doi:10.1136/archdischild-2012-302724.1798

E Okulu, S Arsan, IM Akin, S Alan, A Kilıc, B Atasay. Department of Pediatrics, Division of Neonatology, Ankara University, Ankara, Turkey

Aim To evaluate the clinical outcomes of very-low-birth-weight infants received non-invasive ventilation at delivery room (DR) and NICU.

Methods This prospective cohort study included infants born before 29 weeks', and infants born at 29-30 weeks' who didn't receive antenatal steroid (ANS) from January-2009 to December 2011. During resuscitation, stabilization and transport infants were ventilated with a T-piece. All received $100 \mathrm{mg} / \mathrm{kg}$ surfactant. If respiratory drive was present, infants were extubated to nasal CPAP(NCPAP). The need for MV within the first 3-days, neonatal morbidities, mortality, and duration of hospitalization were assessed.

Results Eighty infants were evaluated. Mean gestational age(GA) and the mean birth weight(BW) of infants were 27.0 \pm 2.1 weeks, and $936.5 \pm 299.1 \mathrm{~g}$, respectively. ANS was given to $27.5 \%$ of the pregnancies. The presence of PPROM and chorioamnionitis were $57.5 \%$ and $34 \%$, respectively. Twenty-three (28.7\%) infants could not be extubated at the DR, and mean GA and BW were lower than the infants who could extubated. Ten(17.5\%) of 57 infants who were on NCPAP initially needed MV during their first 3-days. There wasn't any case with air leak. The incidence of pulmonary hemorrhage, PDA, NEC, İVH, BPD, ROP and mortality were $6.2 \%, 26.2 \%$, $20 \%, 13.8 \%, 10 \%, 10 \%$ and $38.7 \%$, respectively. The duration of respiratory support was 1-720 hours(median: 29 hours). Mean duration of hospitalization was $34.9 \pm 28.4$ days.

Conclusion Our study demonstrated that NCPAP is an effective non-invasive ventilatory strategy. It didn't increase the risk of air leak. The incidences of BPD and ROP in our series were lower than reported in 'NICHD Neonatal Research Network' data.

\section{RESPIRATORY SIGNALS FROM THE EXTERNAL BODY SURFACE IN SPONTANEOUSLY BREATHING PRETERM INFANTS}

doi:10.1136/archdischild-2012-302724.1799

1,2AW Flemmer, ${ }^{2} \mathrm{~L}$ Owen, ${ }^{1} \mathrm{~A}$ Schulze, ${ }^{1} \mathrm{~S}$ Herber-Jonat, ${ }^{2} \mathrm{PG}$ Davis. 'Div. Neonatology, Perinatal Center, Ludwig-Maximilian-University Munich, Munich, Germany; ${ }^{2}$ Neonatal Research, Royal Women's Hospital, Melbourne, VIC, Australia

Background Synchronized noninvasive ventilation (sNIPPV) might be superior to fixed time-cycled modes. Several body-surface respiratory signals have been suggested for noninvasive synchronization. However, the quality and utility of such signals have not been evaluated comprehensively.

Methods Respiratory signals in spontaneously breathing preterm infants ( $n=9$ ) were recorded simultaneously (i) respiratory impedance plethysmography (RIPabd \& RIPchest), (ii) Graseby Capsule (GC), (iii) a strapless piezo sensor (Piezo), and (iv) a fluid-filled esophageal pressure catheter (Pes). A total of 5813 breaths were evaluated. Subsequently, periods of good-quality signals on all recorded channels were analyzed with regard to signal availability, time lag relative to Pes, and variability of signals.

Results In all breaths studied $38 \%$ of breaths showed valid signals in all channels without movement artefacts. Of all signals, RIPabd indicated the onset of inspiration earliest in time relative to the onset of inspiration detected by Pes $(-52 \pm 160 \mathrm{~ms})$. RIPabd-signal was followed by GC-signal $(+10 \pm 177 \mathrm{~ms})$. Both signals had a reasonable variability. The Piezo-signal was very sensitive and prone to large variations $(+70 \pm 1372 \mathrm{~ms}$ compared to Pes). RIPchest indicated inspiration later than RIPabd (+104 $\pm 212 \mathrm{~ms})$.

Conclusion These data indicate that both RIPabd and Graseby Capsule are suitable surface sensors for non-invasive synchronization of NIPPV whereas a Piezo sensor exhibited large variability. Signals from all studied sensors were only suitable for a limited amount of time.

\section{SURVEY OF THE CURRENT USE OF N-IPPV IN SPANISH NEONATAL UNITS}

doi:10.1136/archdischild-2012-302724.1800

C Carrasco Carrasco, Respiratory PathologySurfactant Group of the Spanish Society of Neonatology (Respisurf). Neonatology Unit, Sant Joan de Deu Hospital, Esplugues de Llobregat, Spain

Background nIPPV is widely spread in Spanish neonatal units. Little evidence about the best mode of delivery or the appropriate ventilatory parameters of nIPPV is available.

The Aim of this study was to assess the current use of nIPPV in Spanish neonatal units.

Methods A survey was designed and sent by email to neonatal units. The survey collected information about the devices and the ventilatory parameters used to deliver nIPPV over 2010. The use of synchronisation was also interrogated.

Results 87 out of 115 questionnaires were answered and returned (75.6\%). 71 units used nIPPV (81.6\%). Infant Flow ${ }^{\circledR}$ was the most used device $(48 / 71 ; 67.6 \%)$, followed by conventional ventilators (38/71, 53.5\%). The initial ventilatory parameters depended on the device that was used. When Infant Flow ${ }^{\circledR}$ was used, PIPs were set between 8 and $10 \mathrm{cmH}_{2} \mathrm{O}$, whereas when a conventional ventilator was used, PIPs varied between 8 and $18 \mathrm{cmH}_{2} \mathrm{O}$. In contrast, PEEP, inflation rate and inflation time were set in a more stable range regardless of which device was used. Regarding synchronisation, only $13 / 71$ units $(18.3 \%)$ always used synchronisation, whereas $27 / 71$ units (38\%) only used it in some cases. The pneumatic capsule was most frequently used when synchronisation was provided with a percentage of $52 \%$ (21/40 units).

Conclusion The most used device to apply nIPPV in Spanish neonatal units was a variable flow device. Overall, low PIP and low inflation rates were set at the beginning of this therapy. Synchronisation was scarcely used.

\section{SURVEY OF THE CURRENT USE OF NON INVASIVE VENTILATION IN SPANISH NEONATAL UNITS}

doi:10.1136/archdischild-2012-302724.1801

C Carrasco Carrasco, Respiratory Pathology and Surfactant Group of the Spanish Society of Neonatology (Respisurf). Neonatology Unit, Sant Joan de Deu Hospital, Esplugues de Llobregat, Spain

Background Nasal ways of ventilation are being used to minimize lung damage due to endotracheal ventilation. Both nCPAP and nIPPV are progressively being more used in neonatal units, in spite of the lack of evidence about the best mode of delivery of nasal ventilation or the appropiate ventilatory parameters.

The Aim of this study was to assess the current state of the application of nCPAP and nIPPV in Spanish neonatal units. 